\title{
Analytic stochastic treatment of a nonlinear quantum model with negative diffusion
}

\author{
Roberta Zambrini ${ }^{1}$ and Stephen M. Barnett ${ }^{2}$ \\ ${ }^{1}$ Instituto Mediterráneo de Estudios Avanzados, IMEDEA (CSIC-UIB), Campus Universitat Illes Balears, \\ E-07071 Palma de Mallorca, Spain* \\ ${ }^{2}$ Department of Physics and Applied Physics, University of Strathclyde, 107 Rottenrow, Glasgow G4 ONG, Scotland
}

(Received 11 September 2001; published 2 May 2002)

\begin{abstract}
We apply a proposal of Yuen and Tombesi for treating stochastic problems with negative diffusion to the analytically soluble problem of the single-mode anharmonic oscillator. We find that the associated stochastic realizations include divergent trajectories. It is possible, however, to solve the stochastic problem exactly, but the averaging must be performed with great care.
\end{abstract}

DOI: 10.1103/PhysRevA.65.053810

PACS number(s): 42.50.Ct, 02.50.Ey, 05.40.Ca

\section{INTRODUCTION}

The treatment of even quite simple quantum optical systems can present a significant technical challenge. The description of any isolated system can be given using a density operator, with time evolution governed by the Liouville equation [1]. When the system of interest is not isolated, but can exchange both energy and fluctuations with the surrounding environment, the evolution of the system density operator is governed by a master equation [2]. In particular, master equations provide a practical method to treat such systems, but direct solution of these is not usually possible. It is often possible, particularly for problems involving optical field modes, to map the operator master equation onto a partial differential equation for a quasiprobability distribution. It may be possible to solve this equation or to map it onto an equivalent stochastic process that can be simulated numerically.

Mapping the quantum problem onto a stochastic system relies on a formal similarity between the partial differential equation, obtained from the master equation, and the FokkerPlanck equation associated with Brownian motion. The Fokker-Planck equation for the dynamics of a single field mode or harmonic oscillator is typically of the form

$$
\begin{aligned}
\frac{\partial}{\partial t} \mathcal{W}= & -\frac{\partial}{\partial \alpha} A_{\alpha} \mathcal{W}-\frac{\partial}{\partial \alpha^{*}} A_{\alpha *} \mathcal{W}+\frac{1}{2} \frac{\partial^{2}}{\partial \alpha^{2}} D_{\alpha \alpha} \mathcal{W} \\
& +\frac{1}{2} \frac{\partial^{2}}{\partial \alpha^{* 2}} D_{\alpha * \alpha *} \mathcal{W}+\frac{\partial^{2}}{\partial \alpha \partial \alpha^{*}} D_{\alpha \alpha *} \mathcal{W},
\end{aligned}
$$

where $\mathcal{W}$ is the quasiprobability distribution for the phase space associated with the mode and parametrized by the complex variables $\alpha$ and $\alpha^{*}[2,3]$. The requirement that $\mathcal{W}$ be a real-valued function imposes the conditions that $A_{\alpha^{*}}^{*}$ $=A_{\alpha}, D_{\alpha^{*} \alpha^{*}}=D_{\alpha \alpha}^{*}$, and $D_{\alpha \alpha^{*}}$ is real. This equation can be mapped onto a pair of stochastic differential equations (SDEs) for the phase-space coordinates (also written as $\alpha$ and $\left.\alpha^{*}\right)$ in the form

\footnotetext{
*http://www.imedea.uib.es/PhysDept/
}

$$
\begin{gathered}
\dot{\alpha}=v_{\alpha}+\xi(t), \\
\dot{\alpha}^{*}=v_{\alpha^{*}}+\xi^{*}(t),
\end{gathered}
$$

where $v_{\alpha}$ and $v_{\alpha^{*}}$ are functions of the drift and diffusion coefficients $\left(A_{i}\right.$ and $\left.D_{i j}\right)$ appearing in Eq. (1) and the overdot denotes a derivative with respect to time. The terms $\xi(t)$ and $\xi^{*}(t)$ are stochastic fluctuating terms with correlation functions related to the diffusion coefficients. There is no unique stochastic representation of a given Fokker-Planck equation. In this paper we work with the Stratonovich form of the stochastic integral [4]. A brief discussion of this is given in the Appendix.

Unfortunately, not all problems of interest can be converted into the Fokker-Planck form (1). Systems of interest in quantum nonlinear optics often produce equations for the evolution of quasiprobabilities that have derivatives of higher than second order and it is not known how to treat these within the stochastic formalism. The usual approach is to simply drop these terms to produce "stochastic electrodynamics." It has been shown, however, that this frequently used approximation does not correctly reproduce higherorder correlations such as those predicted to occur in parametric oscillators [5,6]. A second, more subtle, problem is that even when we do obtain an equation of the form (1), it might still not be possible to map this onto SDEs of the form (2) and (3). The difficulty arises when we have negative diffusion, that is, when $D_{\alpha \alpha}<\left|D_{\alpha \alpha}\right|$. With negative diffusion, we are led to SDEs in which $\xi^{*}$ cannot be the complex conjugate of $\xi$ and hence $\alpha^{*}$ will not be the complex conjugate of $\alpha$. It was to resolve problems of this kind that the positive $P$ representation was introduced [3,7-9].

In this paper we consider a proposal by Yuen and Tombesi to convert the evolution equation for the $Q$ quasiprobability into a pair of SDEs $[10,11]$. Their idea is that the correct averages should be obtained by formal application of the Langevin method by simply ignoring the presence of negative diffusion. These authors applied their method to a singlemode evolving under the influence of a quadratic Hamiltonian in the presence of damping and showed that this gave the known evolution for this problem. In this paper we apply the Yuen-Tombesi approach to the more demanding but still analytically soluble problem of the undamped anharmonic 
oscillator [12-14]. This model is known to cause difficulties with stochastic simulations derived from the positive $P$ representation $[3,9,15]$. We find that the Yuen-Tombesi method gives the correct results but that it cannot reliably be applied to numerical simulations of the problem. We trace the origin of this difficulty to the order in which stochastic averages and averages over the initial phase-space distribution have to be performed.

\section{METHOD OF YUEN AND TOMBESI}

The method of Yuen and Tombesi was designed to deal with problems in which the evolution equation for the $Q$ function displays negative diffusion. The $Q$ function for a single field mode or oscillator can be written in a number of forms, the simplest of which is $[2,3,16]$

$$
Q\left(\alpha, \alpha^{*}\right)=\frac{1}{\pi}\langle\alpha|\hat{\rho}| \alpha\rangle
$$

where $\hat{\rho}$ is the density operator for the mode. This distribution can be used to obtain antinormal ordered moments of the annihilation and creation operators by integration over the complex $\alpha$ plane:

$$
\left\langle\hat{a}^{n} \hat{a}^{\dagger m}\right\rangle=\int d^{2} \alpha Q \alpha^{n} \alpha^{* m} .
$$

We consider systems (such as the anharmonic oscillator) in which the evolution equation for the $Q$ function is of the form given in Eq. (1), with negative diffusion. This leads to associated SDEs in which the stochastic variable $\alpha^{*}(t)$ is not the complex conjugate of $\alpha(t)$. As an example, consider an equation in which $D_{\alpha \alpha^{*}}=0$. This necessarily implies negative diffusion associated with $D_{\alpha \alpha}$ and $D_{\alpha * \alpha *}$. We can follow the method outlined in the Appendix to obtain a pair of SDEs that are equivalent to our evolution equation for $Q$ [17]:

$$
\begin{gathered}
\dot{\alpha}=A_{\alpha}-\frac{1}{4} \frac{\partial}{\partial \alpha} D_{\alpha \alpha}+\sqrt{D_{\alpha \alpha}} f_{\alpha}, \\
\dot{\alpha}^{*}=A_{\alpha}^{*}-\frac{1}{4} \frac{\partial}{\partial \alpha^{*}} D_{\alpha * \alpha *}+\sqrt{D_{\alpha * \alpha} *} f_{\alpha^{*}} .
\end{gathered}
$$

It might appear that these equations are mutual complex conjugates but this is not the case as the two Gaussian noise terms are independent and hence do not take complex conjugate values. It follows that we cannot interpret $\alpha$ and $\alpha^{*}$ as mutual complex conjugates. The situation is reminiscent of that found with the positive $P$ representation and we employ the same notation by writing our stochastic variables as $\alpha(t)$ and $\alpha^{+}(t)$ [7]. Anti-normal-ordered expectation values should then correspond to stochastic averages of the corresponding functions of $\alpha$ and $\alpha^{+}$, with $\hat{a}(t)$ replaced by $\alpha(t)$ and $\hat{a}^{\dagger}(t)$ replaced by $\alpha^{+}(t)$.

We can introduce the variables $\alpha$ and $\alpha^{+}$more formally by means of the complex function

$$
\widetilde{Q}\left(\alpha, \alpha^{+}\right)=\frac{1}{\pi}\left\langle 0\left|e^{\alpha^{+} \hat{a}} \hat{\rho} e^{\alpha \hat{a}^{\dagger}}\right| 0\right\rangle e^{-\alpha^{+} \alpha},
$$

which is a function of $\alpha$ and $\alpha^{+}$but not of their complex conjugates. This reduces to the familiar $Q$ function (4) when $\alpha^{+}=\alpha^{*}$. We can convert our master equation for $\hat{\rho}$ into an evolution equation for $\widetilde{Q}$ by making the substitutions

$$
\begin{gathered}
\hat{\rho} \hat{a}^{\dagger} \rightarrow\left(\alpha^{+}+\frac{\partial}{\partial \alpha}\right) \widetilde{Q}, \\
\hat{a}^{\dagger} \hat{\rho} \rightarrow \alpha^{+} \widetilde{Q}, \\
\hat{a} \hat{\rho} \rightarrow\left(\alpha+\frac{\partial}{\partial \alpha^{+}}\right) \widetilde{Q}, \\
\hat{\rho} \hat{a} \rightarrow \alpha \widetilde{Q} .
\end{gathered}
$$

The resulting equation for $\widetilde{Q}$ will be of the same form as that for our $Q$ function with $\alpha^{*}$ replaced by $\alpha^{+}$.

\section{ANHARMONIC OSCILLATOR}

The anharmonic oscillator is one of the simplest analytically solvable models in quantum optics. The Hamiltonian for this model can be written in the form

$$
\hat{H}=\omega\left(\hat{a}^{\dagger} \hat{a}+\frac{1}{2}\right)+\mu\left(\hat{a}^{\dagger} \hat{a}\right)^{2},
$$

where $\omega$ is the natural angular frequency for the mode and we work with units in which $\hbar=1$. The term proportional to $\mu$ is sometimes written in normal order as $\mu \hat{a}^{\dagger} \hat{a} \hat{a}^{\dagger} \hat{a} \hat{a}$. This is the same model but with the $\omega$ changed to $\omega+\mu$. It is convenient to remove the free evolution of the mode and this can be achieved by working in an interaction picture rotating at angular frequency $\omega$. The interaction picture form of the Hamiltonian (10) is

$$
\hat{H}_{I}=\mu\left(\hat{a}^{\dagger} \hat{a}\right)^{2} .
$$

This Hamiltonian has been used in quantum optics as a model for the Kerr nonlinear refractive index. Despite its simplicity, it produces a number of nonclassical effects including squeezing [14] and Schrödinger cat states [13], that is, superpositions of coherent states. The accurate reproduction of these features, especially the cat states, presents a stiff challenge to a stochastic simulation method such as that proposed by Yuen and Tombesi $[10,11]$. The fact that the model is analytically soluble means that we can compare the results of such simulations with exact analytical expressions. We will give an example of this comparison in the following section. In this section we present a brief review of some of the known features of the model.

It is clear from the form of the Hamiltonian that it commutes with the number operator $\hat{a}^{\dagger} \hat{a}$. It follows that the number of excitations (or photons) in the mode will be conserved 
and that the photon number states $|n\rangle$ will be the eigenstates of our interaction Hamiltonian

$$
\hat{H}_{I}|n\rangle=n^{2} \mu|n\rangle .
$$

The corresponding time-evolution operator is

$$
\hat{U}(t)=\exp \left(-i \hat{H}_{I} t\right)=\sum_{n=0}^{\infty}|n\rangle\langle n| e^{-i n^{2} \mu t},
$$

and it follows that the evolution of our oscillator will be periodic with period $2 \pi / \mu$. If we can expand our initial state in terms of the number states, then we can use this result to solve for the time-evolved state in the Schrödinger picture. For example, an initial coherent state $\left|\alpha_{0}\right\rangle$ will evolve to the state

$$
\hat{U}(t)\left|\alpha_{0}\right\rangle=e^{-\left|\alpha_{0}\right|^{2}} \sum_{n=0}^{\infty} \frac{\alpha_{0}^{n}}{\sqrt{n !}} e^{-i n^{2} \mu t}|n\rangle .
$$

This state has a rich structure that can be seen in pictures of the associated quasiprobability distributions $[12,14]$. The state has a simple form at the quarter periods, when it can be written as [13]

$$
\begin{gathered}
\hat{U}[\pi /(2 \mu)]\left|\alpha_{0}\right\rangle=\frac{1-i}{2}\left|\alpha_{0}\right\rangle+\frac{1+i}{2}\left|-\alpha_{0}\right\rangle, \\
\hat{U}[\pi / \mu]\left|\alpha_{0}\right\rangle=\left|-\alpha_{0}\right\rangle, \\
\hat{U}[3 \pi /(2 \mu)]\left|\alpha_{0}\right\rangle=\frac{1+i}{2}\left|\alpha_{0}\right\rangle+\frac{1-i}{2}\left|-\alpha_{0}\right\rangle .
\end{gathered}
$$

The state at one quarter and three quarters of a period is a superposition of the coherent states $\left|\alpha_{0}\right\rangle$ and $\left|-\alpha_{0}\right\rangle$. Such superposition states have interesting nonclassical properties and have been called Schrödinger cat states.

Our stochastic treatment is designed to produce expectation values of operators for the oscillator. These can also be calculated analytically, but this is most easily performed in the Heisenberg interaction picture. The time-evolved annihilation and creation operators are

$$
\begin{aligned}
& \hat{a}(t)=\hat{U}^{\dagger}(t) \hat{a} \hat{U}(t)=e^{-i \mu t} e^{-i 2 \mu t \hat{a}^{\dagger} \hat{a}} \hat{a}, \\
& \hat{a}^{\dagger}(t)=\hat{U}^{\dagger}(t) \hat{a^{\dagger}} \hat{U}(t)=e^{i \mu t} \hat{a}^{\dagger} e^{i 2 \mu t \hat{a}^{\dagger} \hat{a}},
\end{aligned}
$$

where we have written the initial operators as $\hat{a}$ and $\hat{a}^{\dagger}$. It is straightforward to use these expressions to calculate expectation values for functions of $\hat{a}(t)$ and $\hat{a}^{\dagger}(t)$. For example, the expectation value of the annihilation operator for the coherent state $\left|\alpha_{0}\right\rangle$ is

$$
\begin{aligned}
\langle\hat{a}(t)\rangle & =e^{-i \mu t}\left\langle\alpha_{0}\left|e^{-i 2 \mu t \hat{a}^{\dagger} \hat{a}} \hat{a}\right| \alpha_{0}\right\rangle \\
& =e^{-i \mu t} \alpha_{0} \exp \left[\left|\alpha_{0}\right|^{2}\left(e^{-i 2 \mu t}-1\right)\right] .
\end{aligned}
$$

In this expression we have omitted the free evolution in the form of a factor $e^{-i \omega t}$. This corresponds to working in a frame rotating at frequency $\omega$, associated with our choice of interaction picture. All expressions in this paper will be given in this frame. The expectation value of $\hat{a}^{\dagger}(t)$ is the complex conjugate of Eq. (18) and higher-order moments can also be calculated without difficulty.

The evolution equation for the $Q$ function can be written in the form [12]

$$
\begin{aligned}
\frac{\partial}{\partial t} Q= & -i \mu\left[\frac{\partial}{\partial \alpha^{*}}\left(2|\alpha|^{2}-3\right) \alpha^{*} Q-\frac{\partial}{\partial \alpha}\left(2|\alpha|^{2}-3\right) \alpha Q\right. \\
& \left.+\frac{\partial^{2}}{\partial \alpha^{* 2}} \alpha^{* 2} Q-\frac{\partial^{2}}{\partial \alpha^{2}} \alpha^{2} Q\right] .
\end{aligned}
$$

Comparison with Eq. (1) reveals that this equation has negative diffusion $\left(D_{\alpha \alpha^{*}}=0<\left|D_{\alpha \alpha}\right|=2\left|\mu \alpha^{2}\right|\right)$ and hence is a good candidate with which to test the ideas of Yuen and Tombesi. We should emphasize that the partial differential Eq. (19) itself does not present any difficulties in spite of the negative diffusion [18]. Indeed, we can solve this equation directly to give the correct $Q$ function [12].

\section{ANALYTIC STOCHASTIC TREATMENT OF THE ANHARMONIC OSCILLATOR}

We can reexpress the evolution of our $Q$ function, given by Eq. (19) as an equivalent stochastic process using the method outlined in the Appendix. A simple and natural choice is to set $C_{\alpha \alpha^{*}}=0=C_{\alpha^{*} \alpha}$ so that $C_{\alpha \alpha}=\sqrt{i 2 \mu} \alpha$ and $C_{\alpha^{*} \alpha^{*}}=\sqrt{-i 2 \mu} \alpha^{*}$. The evolution equation for our $Q$ function clearly displays negative diffusion and so we write our SDEs in terms of the variables $\alpha$ and $\alpha^{+}$. For the choices described above, our SDEs become

$$
\begin{gathered}
\dot{\alpha}=-i \mu 2\left(\alpha^{+} \alpha-1\right) \alpha+\xi \alpha, \\
\dot{\alpha}^{+}=i \mu 2\left(\alpha^{+} \alpha-1\right) \alpha^{+}+\xi^{+} \alpha^{+},
\end{gathered}
$$

where $\xi$ and $\xi^{+}$are complex, white Gaussian noises with the stochastic averages

$$
\begin{gathered}
\left\langle\xi(t) \xi\left(t^{\prime}\right)\right\rangle_{S}=2 i \mu \delta\left(t-t^{\prime}\right), \\
\left\langle\xi^{+}(t) \xi^{+}\left(t^{\prime}\right)\right\rangle_{S}=-2 i \mu \delta\left(t-t^{\prime}\right), \\
\left\langle\xi^{+}(t) \xi\left(t^{\prime}\right)\right\rangle_{S}=0 .
\end{gathered}
$$

We will require averages both over the stochastic noise realizations and also over the initial quasiprobability distribution. The subscript $S$ identifies the fact that we have carried out the stochastic average. The stochastic averages (22) do not fully determine the forms of the noise terms. It is clear, however, that $\xi^{+}(t)$ cannot be the complex conjugate of $\xi(t)$. It has been suggested that the considerable freedom in choosing the forms of $\xi(t)$ and $\xi^{+}(t)$ can be used to suppress, although not completely remove, stochastic sampling errors in the analogous problem in the positive $P$ representation. The analysis presented in this section is independent of 
this choice of Gaussian noise and hence the freedom to select the forms of $\xi(t)$ and $\xi^{+}(t)$ will not address the problem uncovered.

We require the solution of Eqs. (20) and (21) with the initial conditions $\alpha(0)=\beta$ and $\alpha^{+}(0)=\beta^{*}$. These mean that $\alpha^{+}(0)=\alpha^{*}(0)$ and allow us to use the initial $Q_{0}$ function to perform the average over the initial state. As already noted in Sec. II, the form of the stochastic noise means that $\alpha^{+}(t)$ will not take the value $\alpha^{*}(t)$ in any given realization. The full quantum average will only be obtained by performing an average over the $Q_{0}$ function for the initial state. For the coherent state $\left|\alpha_{0}\right\rangle$ this is

$$
Q_{0}(\beta)=\frac{1}{\pi} e^{-\left|\beta-\alpha_{0}\right|^{2}} .
$$

We denote the average obtained by integrating over $\beta$ by the subscript $Q_{0}$ :

$$
\left\langle F\left(\alpha(t), \alpha^{+}(t)\right)\right\rangle_{Q_{0}}=\int_{-\infty}^{\infty} d^{2} \beta Q(\beta) F\left(\alpha(t), \alpha^{+}(t)\right) .
$$

Quantum expectation values should be obtained on performing both the stochastic average and the average over this $Q_{0}$ function. In particular, the mean value of $\hat{a}$ at time $t$ will be

$$
\langle\hat{a}(t)\rangle=\left\langle\langle\alpha(t)\rangle_{S}\right\rangle_{Q_{0}} .
$$

We have not yet given a prescription for the order, if any, in which these averages must be performed. We will see that this question is of some importance for the solution of the SDEs.

In this section we will calculate the expectation value of the annihilation operator at time $t$ by solving the SDEs (20) and (21). We start by noticing that the combination $\alpha^{+} \alpha$ satisfies the equation

$$
\frac{d}{d t} \alpha^{+} \alpha=\left(\xi+\xi^{+}\right) \alpha^{+} \alpha
$$

The formal solution to this equation is

$$
\alpha^{+}(t) \alpha(t)=\beta^{*} \beta e^{\int_{0}^{t}\left[\xi\left(t^{\prime}\right)+\xi^{+}\left(t^{\prime}\right)\right] d t^{\prime}} .
$$

This already suggests that the stochastic simulation of this problem may run into difficulties. We expect the average obtained from $\alpha^{+}(t) \alpha(t)$ will be $\left\langle\hat{a}(t) \hat{a}^{\dagger}(t)\right\rangle$, which should take the constant value $\left|\alpha_{0}\right|^{2}+1$. The solution (26), however, clearly shows that the stochastic noise will cause $\alpha^{+}(t) \alpha(t)$ to fluctuate away from its initial value in a single realization of the stochastic process. The average is constant but the corresponding variance increases in time. The presence of a complex driving force $\xi+\xi^{+}$means that $\alpha^{+}(t) \alpha(t)$ can acquire any complex value. Nevertheless, we can proceed by inserting our solution (26) into our SDEs (20) and (21). We find that the resulting equations are linear. In particular, the equation for $\alpha(t)$ becomes

$$
\dot{\alpha}(t)=\left[-i 2 \mu\left(|\beta|^{2} e^{\int_{0}^{t}\left[\xi\left(t^{\prime}\right)+\xi^{+}\left(t^{\prime}\right)\right] d t^{\prime}}-1\right)+\xi(t)\right] \alpha(t),
$$

the solution of which is

$$
\begin{aligned}
\alpha(t)= & \beta \exp \left\{\int _ { 0 } ^ { t } \left[-i 2 \mu\left(|\beta|^{2} e^{t_{0}^{t^{\prime}}\left[\xi\left(t^{\prime \prime}\right)+\xi^{+}\left(t^{\prime \prime}\right)\right] d t^{\prime \prime}}-1\right)\right.\right. \\
& \left.\left.+\xi\left(t^{\prime}\right)\right] d t^{\prime}\right\} .
\end{aligned}
$$

Similar expressions have been given for the same model treated in the positive $P$ representation [15]. The average of this quantity should be the expectation value of $\hat{a}(t)$. Let us start by performing the stochastic average. This can be achieved most readily by expanding the outer exponential in powers of $|\beta|^{2}$ :

$$
\begin{aligned}
\langle\alpha(t)\rangle_{S}= & \beta e^{i 2 \mu t}\left\langlee ^ { \int _ { 0 } ^ { t } \xi ( t ^ { \prime } ) d t ^ { \prime } } \left[ 1-i 2 \mu|\beta|^{2}\right.\right. \\
& \times \int_{0}^{t} e^{f_{0}^{t^{\prime}}\left[\xi\left(t^{\prime \prime}\right)+\xi^{+}\left(t^{\prime \prime}\right)\right] d t^{\prime \prime}} d t^{\prime} \\
& -2 \mu^{2}|\beta|^{4} \int_{0}^{t} d t^{\prime} \int_{0}^{t} d t^{\prime \prime} e^{f_{0}^{t^{\prime}}\left[\xi(s)+\xi^{+}(s)\right] d s} \\
& \left.\left.\times e^{f_{0}^{t^{\prime \prime}}\left[\xi\left(s^{\prime}\right)+\xi^{+}\left(s^{\prime}\right)\right] d s^{\prime}}+\cdots\right]\right\rangle_{S} .
\end{aligned}
$$

Here we have made explicit use of the Gaussian nature of our stochastic noise in evaluating the averages of exponential functions of the noise. We can evaluate the average of each term in turn. The order zero and order 1 terms are

$$
\begin{gathered}
\left\langle e^{\int_{0}^{t} \xi\left(t^{\prime}\right) d t^{\prime}}\right\rangle_{S}=e^{i \mu t}, \\
-i 2 \mu|\beta|^{2}\left\langle\int_{0}^{t} d t^{\prime} e^{\int_{0}^{t^{\prime}}\left[2 \xi(s)+\xi^{+}(s)\right] d s} e^{\int_{t^{\prime}}^{t} \xi\left(s^{\prime}\right) d s^{\prime}}\right\rangle_{S} \\
=-i 2 \mu|\beta|^{2} \int_{0}^{t} d t^{\prime} e^{4 i \mu t^{\prime}} e^{-i \mu t^{\prime}} e^{i \mu\left(t-t^{\prime}\right)} \\
=-|\beta|^{2} e^{i \mu t}\left(e^{2 i \mu t}-1\right) .
\end{gathered}
$$

It is straightforward to show that the stochastic average of the term of order $|\beta|^{2 n}$ is $(-1)^{n}|\beta|^{2 n} e^{i \mu t}\left(e^{2 i \mu t}-1\right)^{n} / n$ ! It is tempting to resum the series in Eq. (28) to give

$$
\langle\alpha(t)\rangle_{S}=\beta e^{i 3 \mu t} \exp \left[|\beta|^{2}\left(1-e^{i 2 \mu t}\right)\right] .
$$

Let us see the consequences of this resumming by completing our calculation of the expectation value of $\hat{a}(t)$ with the average over $\beta$. This procedure leads to the expression

$\left\langle\langle\alpha(t)\rangle_{S}\right\rangle_{Q_{0}}=\frac{1}{\pi} \int d^{2} \beta e^{-\left|\beta-\alpha_{0}\right|^{2}} \beta e^{i 3 \mu t} \exp \left[|\beta|^{2}\left(1-e^{i 2 \mu t}\right)\right]$.

Inspection of the integrand reveals a problem. It is clear that the integrand is unbounded (and the integral undefined) for times $t$ such that $\cos (2 \mu t) \leqslant 0$. It is interesting to note that this includes the times $\pi /(2 \mu)$ and $3 \pi /(2 \mu)$ at which the anharmonic oscillator evolves into the Schrödinger cat states given in Eqs. (15). The problem is that we have assumed that it is 
acceptable to perform the stochastic average before performing the average over initial conditions. In fact, this is not the case and we should perform the $Q_{0}$ average first. We can see this by evaluating the average over the $Q_{0}$ function before resumming the series in our stochastic average given in Eq. (28). This gives the final average value

$$
\begin{aligned}
\left\langle\langle\alpha(t)\rangle_{Q_{0}}\right\rangle_{S}= & e^{i 3 \mu t} \sum_{n=0}^{\infty} \frac{\left(1-e^{i 2 \mu t}\right)^{n}}{n !} \\
& \times \int \frac{d^{2} \beta}{\pi} \beta^{n+1} \beta^{* n} e^{-\left|\beta-\alpha_{0}\right|^{2}} \\
= & e^{i 3 \mu t} \sum_{n=0}^{\infty}\left(1-e^{i 2 \mu t}\right)^{n} \sum_{l=0}^{n} \frac{(n+1) !}{l !(l+1) !(n-l) !} \\
& \times \alpha_{0}^{l+1} \alpha_{0}^{* l} \\
= & e^{i 3 \mu t} \sum_{l=0}^{\infty} \frac{\left|\alpha_{0}\right|^{2 l}}{(l+1) !} \sum_{n=l}^{\infty}\left(1-e^{i 2 \mu t}\right)^{n} \frac{(n+1) !}{(n-l) ! l !} \\
= & e^{-i \mu t} \alpha_{0} \exp \left(\left|\alpha_{0}\right|^{2}\left(e^{-i 2 \mu t}-1\right)\right),
\end{aligned}
$$

which we recognize as the correct answer given in Eq. (18). Other moments can be obtained in the same manner.

We can see the origin of the incorrect stochastic average given in Eq. (31) by considering the form of the annihilation operator in the Heisenberg picture, Eq. (16), which we can also write in the form

$$
\hat{a}(t)=e^{3 i \mu t} \hat{a} e^{-i 2 \mu t \hat{a}^{\dagger} \hat{a}}=e^{3 i \mu t} \hat{a}: e^{\left(1-e^{i 2 \mu t}\right) \hat{a} \hat{a}^{\dagger}}:
$$

where : $:$ denotes antinormal ordering and we have used the antinormal ordering theorem for the exponential of $\hat{a}^{\dagger} \hat{a}$ [2]. We note that this becomes the expression (31) obtained by performing the stochastic average, if we identify $\hat{a}$ and $\hat{a}^{\dagger}$ with $\beta$ and $\beta^{*}$, respectively. We have written Eq. (33) in antinormal order because the $Q$ function gives antinormally ordered moments. If we use this expression to calculate the expectation value of $\hat{a}(t)$, for our initial coherent state, then we find

$$
\langle\hat{a}(t)\rangle=e^{3 i \mu t}\left\langle\alpha_{0}\left|\hat{a} \vdots \exp \left[\left(1-e^{i 2 \mu t}\right) \hat{a} \hat{a}^{\dagger}\right]:\right| \alpha_{0}\right\rangle .
$$

We can, of course, evaluate this expectation value by putting the operator into normal ordered form and using the fact that the coherent states are right eigenstates of the annihilation operator. Our aim, however, is to investigate the problems with the stochastic average associated with simulations designed to reproduce antinormal ordered averages. We can work with the antinormal ordered form in Eq. (34) by expanding the exponential as a Taylor series and inserting the identity in the form of an integral over the coherent states $|\beta\rangle[2]$ :

$$
\begin{aligned}
\langle\hat{a}(t)\rangle= & e^{3 i \mu t}\left\langle\alpha_{0}\left|\sum_{n=0}^{\infty} \frac{\left(1-e^{i 2 \mu t}\right)^{n}}{n !} \hat{a}^{n+1} \int \frac{d^{2} \beta}{\pi}\right| \beta\right\rangle \\
& \times\left\langle\beta\left|\hat{a}^{\dagger n}\right| \alpha_{0}\right\rangle \\
= & e^{3 i \mu t} \sum_{n=0}^{\infty} \frac{\left(1-e^{i 2 \mu t}\right)^{n}}{n !} \int \frac{d^{2} \beta}{\pi} \beta|\beta|^{2 n} e^{-\left|\beta-\alpha_{0}\right|^{2}} .
\end{aligned}
$$

Clearly, it would be wrong to evaluate the summation before carrying out the integral. Evaluating the integral first corresponds, in our stochastic treatment, to averaging over initial conditions before performing the stochastic average, and gives the correct result.

It is interesting to note that there is a strong similarity between the SDEs discussed here and those found for the anharmonic oscillator in the positive $P$ representation. Indeed, if we write equations for $\alpha e^{-i \mu t}$ and $\alpha^{+} e^{i \mu t}$, then we recover the equations discussed by Plimak et al. [15]. An important difference, however, is that the diffusion for the positive $P$ representation occurs with the opposite sign from that for the $Q$ function. This means that the stochastic averages (22) have opposite signs when applied to the positive $P$ representation. We can use the methods described in this section to obtain the expectation value of $\hat{a}(t)$ in the positive $P$ representation. The stochastic average gives $\langle\alpha(t)\rangle_{S}$ $=\beta e^{-i \mu t} \exp \left[|\beta|^{2}\left(e^{-i 2 \mu t}-1\right)\right]$. Performing the average of this over a $\delta$-function positive $P$ distribution, peaked at $\beta=\alpha_{0}$ $=\beta^{+*}$, gives the correct result (18). The positive $P$ representation is associated with operator moments in normal order and this seems to be the reason for the well-behaved form of the stochastic averages for initial coherent states.

\section{STOCHASTIC SIMULATION OF THE ANHARMONIC OSCILLATOR}

In this section we present results of numerical simulations [19] of the stochastic process $\alpha(t)$ given in Eq. (27). Our simulations were performed using two discrete Gaussian processes $\eta_{l}, \eta_{l}^{+}$of the form

$$
\eta_{l}=\int_{\Delta t} d t^{\prime} \xi\left(t^{\prime}\right), \quad \eta_{l}^{+}=\int_{\Delta t} d t^{\prime} \xi^{+}\left(t^{\prime}\right)
$$

where $t=l \Delta t$. In this way $\left\langle\eta_{l} \eta_{l^{\prime}}\right\rangle=2 i \mu \Delta t \delta_{l l^{\prime}}$ and $\left\langle\eta_{l}^{+} \eta_{l^{\prime}}^{+}\right\rangle=-2 i \mu \Delta t \delta_{l l^{\prime}}$. We note that the relations (22) do not completely specify the two independent complex white noises. As recently shown in [15] the degree of freedom in the choice of the noise could be used to improve the results of the numerical simulation by choosing the stochastic processes $\xi$ and $\xi^{+}$so as to inhibit (but not completely suppress) the fast growth of $\alpha(t)$. In this paper, however, we have considered only the forms $\xi=\sqrt{2 i \mu} \phi$ and $\xi^{+}=\sqrt{-2 i \mu} \phi^{+}$ with $\phi$ and $\phi^{+}$being real white noises.

Each stochastic realization must start from a single point in phase space. For this reason, the analysis of the preceding section leads us to conclude that diverging trajectories, exploring large values of $|\alpha|$, are inevitable. These divergences 


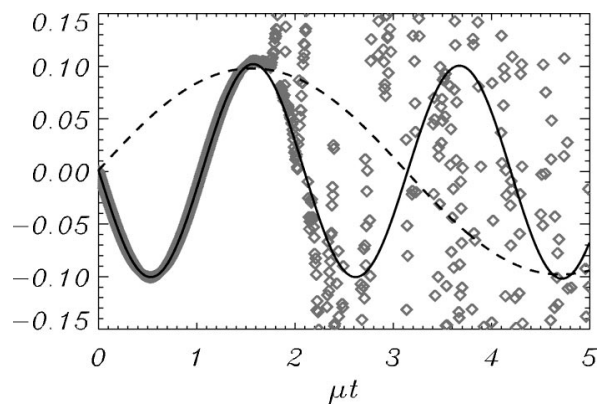

FIG. 1. Time evolution of the stochastic average $\operatorname{Re}\langle\alpha(t)\rangle_{S}$, using 50000 trajectories and starting from $\beta=0.001+i 0.1$. The diamonds represent numerical values. The continuous line represents the analytical result for $\langle\alpha(t)\rangle_{S}$. The dashed line represents $\left\langle\langle\alpha(t)\rangle_{Q_{0}}\right\rangle_{S}$ for the initial coherent state $\left|\alpha_{0}\right\rangle$ with $\alpha_{0}=0.001$ $+i 0.1$.

are responsible for the unbounded average obtained by performing the stochastic average before the average over the initial $Q$ distribution. Each of our simulations starts at a point $\alpha(0)=\alpha^{+*}(0)=\beta$. Naturally, the average over the initial $Q$ distribution requires stochastic realizations for a range of values of $\beta$, weighted by the distribution (23). Consideration of a single value of $\beta$, however, suffices to illustrate the divergences associated with individual trajectories. We observe, in each case, a divergence after some time. We can see the origin of these divergences in the SDEs (20) and (21); the complex variables $\alpha$ and $\alpha^{+}$are not constrained to be complex conjugate quantities and so, in any given realization, the combination $\alpha^{+} \alpha$ can acquire an imaginary part. This leads to exponential growth of $\alpha$ or $\alpha^{+}$. The time at which this divergence appears varies between realizations and also depends on the initial conditions. In particular, the divergence appears earlier for larger values of $|\beta|^{2}$. This is because of the exponential dependence of $\alpha(t)$ on $|\beta|^{2}$ as seen in Eq. (27).

If we select a sufficiently small value of $\beta$ and perform an average over a large number of trajectories then we find a result that is, for short times, in good agreement with the analytical average Eq. (31). In Fig. 1 we have plotted the time evolution of $\operatorname{Re}\langle\alpha(t)\rangle_{S}$, obtained by considering 50000 trajectories, starting from the initial condition $\beta$ $=0.001+i 0.1$ (diamonds). For comparison the analytical expression for the stochastic average is represented by a continuous line. At very short times, we observe a near perfect agreement between the numerical results and the analytical expression. At longer times, this agreement is lost because of the divergence induced by the independent stochastic noises.

The trajectories start from a single point in phase space. This corresponds to selecting, in each simulation, a $\delta$ - function phase-space probability distribution. Such a narrow distribution for the $Q_{0}$ distribution does not correspond to any physically allowed state [3]. Indeed, the evolution obtained from the Fokker-Planck equation for such an initial condition is highly singular. It is this behavior that is reflected in the divergent numerical simulations. Figure 2 depicts the numerically obtained value of $\langle\alpha(t)\rangle_{S}$. We see that this aver-

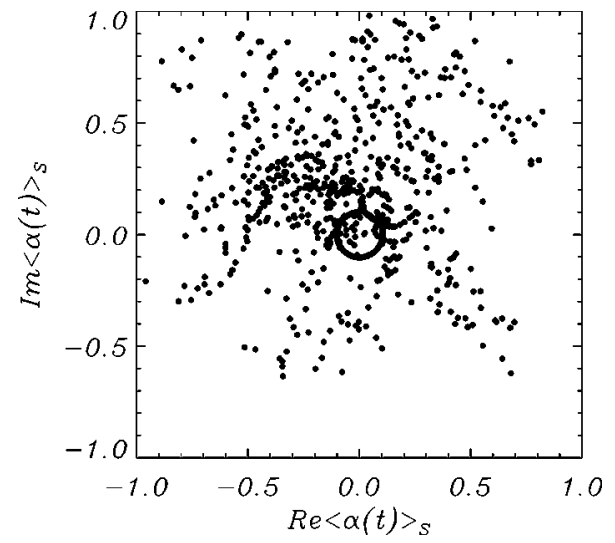

FIG. 2. Phase-space plot of the numerical average $\langle\alpha(t)\rangle_{S}$, the real part of which is shown in Fig. 1. The points represent numerical values at different times. The circle represents the analytical result for $\langle\alpha(t)\rangle_{S}$.

age explores an extended region of the complex plane. The analytical average Eq. (31), is represented by the small circle.

The relationship between the time at which trajectories diverge and the initial condition $(\beta)$ means that an ensemble of trajectories starting from a range of different initial conditions will rapidly produce divergences. For this reason the analytical result (18) cannot be reproduced numerically in any straightforward manner.

\section{CONCLUSION}

In conclusion, we have considered a proposal of Yuen and Tombesi [10] to give a stochastic representation of a FokkerPlanck equation with negative diffusion for the $Q$ representation. We have shown that the correct analytical moments for an anharmonic oscillator (associated with a nonlinear $\chi^{(3)}$ process) can be obtained from the SDEs. These results, however, are highly sensitive to the order in which averages over the stochastic realizations and over the distribution of the initial conditions are performed. It is clear that more sophisticated techniques are required for stochastic simulation of the problem. Recent work suggests that the effects of divergences can be significantly suppressed but not yet eliminated $[15,20]$.

The system studied in this paper is highly idealized. We could include the effects of loss and expect that these will improve the stability of the numerical results. Such an improvement has been noted in studies of positive $P$ [9]. It is possible, however, that there are other interesting systems that are less sensitive to the noise and for these, stochastic simulations using the Yuen-Tombesi method may prove to be a useful technique. Possible systems for study in quantum optics include the optical parametric oscillator and second harmonic generation, which could be successfully studied with this approach. Our preliminary studies suggest that there are regimes of operation, including the threshold, in which the probability for a divergent trajectory to occur is very small. In this case, numerical simulation does give stable results. We will return to this topic elsewhere. 


\section{ACKNOWLEDGMENTS}

We are grateful to Pere Colet, Emilio Hernandez Garcia, Gian-Luca Oppo, Maxi San Miguel, and Raul Toral for their suggestions and encouragement. This work was supported through the European Commission projects QSTRUCT (Project No. ERB FMRX-CT96-0077) and QUANTIM (Project No. IST-2000-26019). S.M.B. thanks the Royal Society of Edinburgh and the Scottish Executive Education and Lifelong Learning Department for financial support.

\section{APPENDIX}

In this appendix we present a brief discussion of the link between a given Fokker-Planck equation and an equivalent stochastic system (a more complete account can be found in $[4,21]$ ). As we have already noted, the Fokker-Planck equation does not correspond to a unique stochastic system and so it is natural to start with a stochastic system. Consider the pair of (Langevin) SDEs

$$
\begin{gathered}
\frac{\partial \alpha}{\partial t}=B_{\alpha}+C_{\alpha \alpha} f_{\alpha}(t)+C_{\alpha \alpha^{*}} f_{\alpha *}(t), \\
\frac{\partial \alpha^{*}}{\partial t}=B_{\alpha^{*}}+C_{\alpha * \alpha *} f_{\alpha *}(t)+C_{\alpha^{*} \alpha} f_{\alpha}(t),
\end{gathered}
$$

with white Gaussian noise terms $f_{i}$ defined to have zero average and second moments of the form

$$
\left\langle f_{i}(t) f_{j}\left(t^{\prime}\right)\right\rangle=\delta_{i j} \delta\left(t-t^{\prime}\right)
$$

and the subscripts $i, j$ denoting $\alpha$ and $\alpha^{*}$.

The formal solution of Eqs. (A1) and (A2) is

$$
\begin{aligned}
\alpha(t)= & \alpha(0)+\int_{0}^{t} d t^{\prime} B_{\alpha}\left(t^{\prime}\right)+\int_{0}^{t} C_{\alpha \alpha} d W\left(t^{\prime}\right) \\
& +\int_{0}^{t} C_{\alpha \alpha^{*}} d W_{*}\left(t^{\prime}\right), \\
\alpha^{*}(t)= & \alpha^{*}(0)+\int_{0}^{t} d t^{\prime} B_{\alpha}^{*}\left(t^{\prime}\right)+\int_{0}^{t} C_{\alpha^{*} \alpha} d W\left(t^{\prime}\right) \\
& +\int_{0}^{t} C_{\alpha * \alpha^{*}} d W_{*}\left(t^{\prime}\right),
\end{aligned}
$$

where we have introduced the Wiener processes $d W(t)$ $=f_{\alpha}(t) d t$ and $d W_{*}(t)=f_{\alpha *}(t) d t$.

In order to use these stochastic processes, we need to give a prescription for carrying out the stochastic integrals over the Wiener processes. In this paper, we choose the Stratonovich interpretation of the stochastic integral in which

$$
\begin{aligned}
\int_{0}^{t} g\left(\alpha, \alpha^{*}\right) d W\left(t^{\prime}\right) \\
=\sum_{i}\left[W\left(t_{i}\right)-W\left(t_{i-1}\right)\right] \\
\quad \times g\left(\frac{\alpha\left(t_{i}\right)+\alpha\left(t_{i-1}\right)}{2}, \frac{\alpha^{*}\left(t_{i}\right)+\alpha^{*}\left(t_{i-1}\right)}{2}\right) .
\end{aligned}
$$

The reason for this choice, instead of the Ito interpretation, is that we will be constructing analytical averages over the stochastic process and the Stratonovich formalism allows us to use the familiar rules of calculus.

From the Langevin equations it is possible to obtain a unique Fokker-Planck equation for the probability distribution $\mathcal{W}\left(\alpha(t), \alpha^{*}(t), t\right)$. If we consider the trajectory obtained in a single realization of the stochastic process $\vec{f}$ $=\left(f_{\alpha}, f_{\alpha^{*}}\right)$ and start from the initial value $\vec{\alpha}(0)$ $=\left(\alpha(0), \alpha^{*}(0)\right)$, then the solution at time $t$ is completely determined and the probability distribution for it is the $\quad \delta \quad$ function $\quad \delta(\alpha-\alpha(t ; \vec{\alpha}(0) ;[\vec{f}(t)])) \delta\left(\alpha^{*}\right.$ $\left.-\alpha^{*}(t ; \vec{\alpha}(0) ;[\vec{f}(t)])\right)$. Considering a set of initial conditions $\overrightarrow{\alpha_{0}}$, distributed according some initial distribution $p_{0}$ $=p(\vec{\alpha}(0) ; 0)$, we can obtain the shape of the distribution at time $t$ :

$$
\begin{aligned}
p(\vec{\alpha}, t ;[\vec{f}(t)])= & \langle\delta(\alpha-\alpha(t ; \vec{\alpha}(0) ;[\vec{f}(t)])) \\
& \left.\times \delta\left(\alpha^{*}-\alpha^{*}(t ; \vec{\alpha}(0) ;[\vec{f}(t)])\right)\right\rangle_{p_{0}},
\end{aligned}
$$

where the subscript $p_{0}$ denotes an average over the initial probability distribution. The quantity $p$ satisfies the conservation equation

$$
\frac{\partial}{\partial t} p+\frac{\partial}{\partial \alpha}(\dot{\alpha} p)+\frac{\partial}{\partial \alpha^{*}}\left(\dot{\alpha}^{*} p\right)=0
$$

The complete probability distribution is obtained by also averaging over the stochastic trajectories obtained with different noise realizations, denoted by the subscript $[\vec{f}(t)]$ :

$$
\mathcal{W}\left(\alpha, \alpha^{*}, t\right)=\left\langle p\left(\alpha, \alpha^{*}, t ;[\vec{f}(t)]\right)\right\rangle_{[\vec{f}(t)]} .
$$

The time evolution for the distribution $\mathcal{W}$ can be obtained using the continuity equation and gives $[4,22]$

$$
\frac{\partial}{\partial t} \mathcal{W}=-\frac{\partial}{\partial \alpha} B_{\alpha} \mathcal{W}-\frac{\partial}{\partial \alpha^{*}} B_{\alpha} * \mathcal{W}+\frac{1}{2} \sum_{i j l}\left[\partial_{i} C_{i j} \partial_{l} C_{l j}\right] \mathcal{W},
$$

where the subscripts $i, j, k$ again denote $\alpha$ and $\alpha^{*}$. If we compare this form of the Fokker-Planck equation with Eq. (1), then we obtain the correspondences

$$
\begin{gathered}
D_{\alpha \alpha}=C_{\alpha \alpha}^{2}+C_{\alpha \alpha^{*}}^{2}, \\
D_{\alpha * \alpha *}=C_{\alpha * \alpha *}^{2}+C_{\alpha * \alpha}^{2}, \\
D_{\alpha \alpha *}=C_{\alpha \alpha} C_{\alpha * \alpha}+C_{\alpha * \alpha *} C_{\alpha \alpha^{*}},
\end{gathered}
$$




$$
\begin{aligned}
A_{\alpha}= & B_{\alpha}+\frac{1}{2}\left[\left(\frac{\partial}{\partial \alpha} C_{\alpha \alpha}\right) C_{\alpha \alpha}+\left(\frac{\partial}{\partial \alpha^{*}} C_{\alpha \alpha}\right) C_{\alpha^{*} \alpha}\right. \\
& \left.+\left(\frac{\partial}{\partial \alpha} C_{\alpha \alpha^{*}}\right) C_{\alpha \alpha^{*}}+\left(\frac{\partial}{\partial \alpha^{*}} C_{\alpha \alpha^{*}}\right) C_{\alpha^{*} \alpha^{*}}\right], \\
A_{\alpha^{*}}= & B_{\alpha^{*}}+\frac{1}{2}\left[\left(\frac{\partial}{\partial \alpha} C_{\alpha^{*} \alpha}\right) C_{\alpha \alpha^{*}}+\left(\frac{\partial}{\partial \alpha^{*}} C_{\alpha^{*} \alpha}\right) C_{\alpha^{*} \alpha}\right. \\
& \left.+\left(\frac{\partial}{\partial \alpha} C_{\alpha^{*} \alpha^{*}}\right) C_{\alpha \alpha^{*}}+\left(\frac{\partial}{\partial \alpha^{*}} C_{\alpha^{*} \alpha^{*}}\right) C_{\alpha^{*} \alpha^{*}}\right] .
\end{aligned}
$$

Note that these equations do not give unique forms for the $B$ and $C$ functions. This is a consequence of the lack of a unique stochastic representation for a given Fokker-Planck equation.

If our stochastic variables $\alpha$ and $\alpha^{*}$ are to be complex conjugate quantities, then it follows from Eqs. (A1) and (A2) that $B_{\alpha *}^{*}=B_{\alpha}, C_{\alpha \alpha}^{*}=C_{\alpha * \alpha}$, and $C_{\alpha \alpha^{*}}^{*}=C_{\alpha^{*} \alpha^{*}}$. These conditions necessarily imply positive diffusion as, from (A9)(A11), $D_{\alpha \alpha^{*}}>\left|D_{\alpha \alpha}\right|$. It follows that the stochastic variables cannot be complex conjugate quantities when we have negative diffusion. In order to avoid possible confusion, we replace the stochastic variable $\alpha^{*}(t)$ by $\alpha^{+}(t)$ whenever there is negative diffusion.
[1] K. Huang, Statistical Mechanics, 2nd ed. (John Wiley and Sons, New York, 1987).

[2] S. M. Barnett and P. Radmore, Methods in Theoretical Quantum Optics (Oxford University Press, Oxford, 1997).

[3] C. W. Gardiner and P. Zoller, Quantum Noise, 2nd ed. (Springer-Verlag, Berlin, 2000).

[4] C. W. Gardiner, Handbook of Stochastic Processes (SpringerVerlag, Berlin, 1985).

[5] P.D. Drummond and P. Kinsler, Quantum Semiclassic. Opt. 7, 727 (1985).

[6] P. Kinsler, Phys. Rev. A 53, 2000 (1996).

[7] P.D. Drummond and C.W. Gardiner, J. Phys. A 13, 2353 (1980).

[8] P.D. Drummond, C.W. Gardiner, and D.F. Walls, Phys. Rev. A 24, 914 (1981).

[9] A. Gilchrist, C.W. Gardiner, and P.D. Drummond, Phys. Rev. A 55, 3014 (1997).

[10] H.P. Yuen and P. Tombesi, Opt. Commun. 59, 155 (1986).

[11] P. Tombesi, Phys. Lett. A 132, 241 (1988).

[12] G.J. Milburn, Phys. Rev. A 33, 674 (1986).
[13] B. Yurke and D. Stoler, Phys. Rev. Lett. 57, 13 (1986).

[14] R. Tanás, A. Miranowicz, and S. Kielich, Phys. Rev. A 43, 4014 (1991).

[15] L.I. Plimak, M.K. Olsen, and M.J. Collett, Phys. Rev. A 64, 025801 (2001).

[16] This definition differs by a factor of $\pi$ from that used by Milburn [12].

[17] These are obtained from the equations given in the Appendix by the choosing $C_{\alpha \alpha^{*}}=0=C_{\alpha * \alpha}$.

[18] K. Vogel and H. Risken, Phys. Rev. A 39, 4675 (1989).

[19] Numerical simulation are performed using the Gaussian random number generator proposed by R. Toral and A. Chakrabarti, Comput. Phys. Commun. 74, 327 (1993).

[20] I. Carusotto, Y. Castin, and J. Dalibard, Phys. Rev. A 63, 023606 (2001).

[21] M. San Miguel and R. Toral, in Instabilities and Nonequilibrium Structures VI, edited by E. Tirapegui, J. Martnez, and R. Tiemann (Kluwer Academic, Dordrecht, 2000), p. 35.

[22] J.M. Sancho, M. San Miguel, S.L. Katz, and J.D. Gunton, Phys. Rev. A 26, 1589 (1982). 